\title{
Calculating Free Energy Changes in Continuum Solvation Models
}

Junming $\mathrm{Ho}^{1,2} *$ and Mehmed Z. Ertem ${ }^{3}$

${ }^{1}$ Agency for Science, Technology and Research, Institute of High Performance

Computing, 1 Fusionopolis Way, \#16-16, Connexis, Singapore 138632. ${ }^{2}$ Department

of Chemistry, Yale University, P. O. Box 208107, New Haven CT 06520, USA.

${ }^{3}$ Chemistry Department, Brookhaven National Laboratory, Upton, NY 11973, USA

Email: hojm@ihpc.a-star.edu.sg

\section{SUPPORTING INFORMATION}

\begin{tabular}{|c|c|c|}
\hline Table & Contents & Page \\
\hline S1 & Worked example $-\mathrm{p} K_{\mathrm{a}}$ calculation of methanol (TC approach) & S2 \\
\hline S2 & Worked example $-\mathrm{p} K_{\mathrm{a}}$ calculation of methanol (Direct approach) & S4 \\
\hline S3 & Calculated and experimental aqueous $\mathrm{p} K_{\mathrm{a}}$ values & S5 \\
\hline S4 & Calculated and experimental reduction potentials & S10 \\
\hline S5-7 & Calculated aqueous $\mathrm{p} K_{\mathrm{a}}$ values using cluster-continuum scheme & S13 \\
\hline S8 & Calculated and experimental free energies of enolization & S19 \\
\hline S9 & Calculated free energies of hydrogen atom and chlorine atom transfer & $\mathrm{S} 21$ \\
\hline $\mathrm{S} 10$ & $\begin{array}{l}\text { Calculated and experimental Gibbs free energy barriers for } 18 \text { Diels Alder } \\
\text { reactions }\end{array}$ & S22 \\
\hline $\mathrm{S} 11$ & $\begin{array}{l}\text { Calculated and experimental Gibbs free energy barriers for } 21 \text { radical } \\
\text { reactions }\end{array}$ & $\mathrm{S} 25$ \\
\hline $\mathrm{S} 12$ & $\begin{array}{l}\text { Calculated and experimental Gibbs free energy barriers for } 45 \text { ionic } \\
\text { reactions }\end{array}$ & S27 \\
\hline
\end{tabular}


S1. Worked example: Procedure for calculating the aqueous $\mathrm{pKa}$ of methanol (at $298 \mathrm{~K}$ ) via a thermodynamic cycle and the SMD-M062X solvation model.

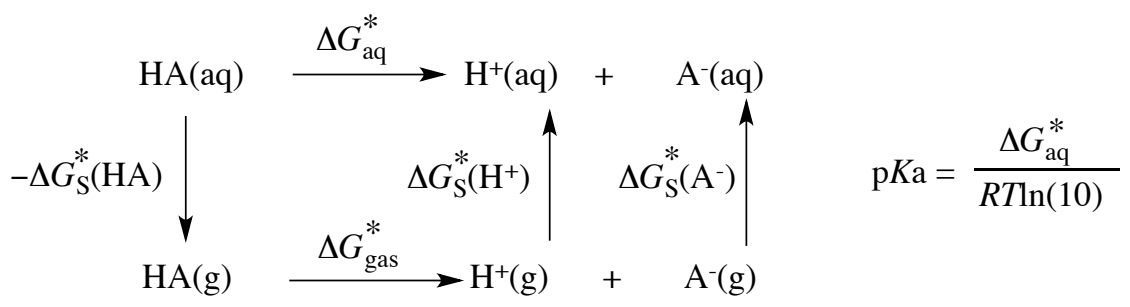

\section{Thermodynamic cycle approach}

$$
\begin{aligned}
& \Delta G_{\text {soln }}^{*}(\mathrm{TC})=\Delta G_{\text {gas }}^{*}+\Delta G_{\mathrm{S}}^{*}\left(\mathrm{~A}^{-}\right)+G_{\mathrm{S}}^{*}\left(\mathrm{H}^{+}\right)-\Delta G_{\mathrm{S}}^{*}(\mathrm{HA}) \\
& \Delta G_{\text {soln }}^{*}(\mathrm{TC})=\Delta E_{\text {soln }}^{[\mathrm{L}]}+\Delta G_{\text {corr, soln }}^{\mathrm{o}[\mathrm{L}]}+\Delta E_{\text {gas }}^{[\mathrm{H}]}-\Delta E_{\text {gas }}^{[\mathrm{L}]}+G_{\text {soln }}^{\mathrm{o}}\left(\mathrm{H}^{+}\right)+\Delta G^{\mathrm{o} \rightarrow *} \\
& \Delta E_{\text {soln }}^{\mathrm{L}}=E_{\text {soln }}^{\mathrm{L}}\left(A^{-}\right)-E_{\text {soln }}^{\mathrm{L}}(H A) \\
& \Delta E_{\text {gas }}^{\mathrm{L}}=E_{\text {gas }}^{\mathrm{L}}\left(A^{-}\right)-E_{\text {gas }}^{\mathrm{L}}(H A) \\
& \Delta G_{\text {corr }}^{\text {soln }}=G_{\text {corr }}^{\text {soln }, \mathrm{L}}\left(A^{-}\right)-G_{\text {corr }}^{\text {soln }, \mathrm{L}}(H A)
\end{aligned}
$$

\begin{tabular}{|c|c|c|c|}
\hline & Methanol & Methoxide & $\mathrm{H}^{+}$ \\
\hline $\mathrm{E}_{\mathrm{gas}}\left[(\mathrm{RO}) \mathrm{CCSD}(\mathrm{T}) / 6-31+\mathrm{G}^{*}\right] /$ Hartree & -115.3862641 & -114.7684752 & \\
\hline $\mathrm{E}_{\mathrm{gas}}[(\mathrm{RO}) \mathrm{MP} 2 / \mathrm{GTMP} 2 \mathrm{Large} /$ Hartree & -115.5079947 & -114.8869668 & \\
\hline $\mathrm{E}_{\mathrm{gas}}\left[(\mathrm{RO}) \mathrm{MP} 2 / 6-31+\mathrm{G}^{*}\right] /$ Hartree & -115.3576499 & -114.7441755 & \\
\hline Higher-level correction / Hartree & -0.06589100 & -0.06589100 & \\
\hline $\mathrm{E}_{\mathrm{gas}}[\mathrm{G} 3(\mathrm{MP} 2)-\mathrm{RAD}(+)] /$ Hartree & -115.6024999 & -114.9771575 & \\
\hline $\mathrm{E}_{\mathrm{gas}}\left(\mathrm{M} 06-2 \mathrm{X} / 6-31+\mathrm{G}^{*}\right) /$ Hartree & -115.6628908 & -115.045887 & \\
\hline $\mathrm{E}_{\mathrm{aq}}\left(\mathrm{M} 06-2 \mathrm{X} / 6-31+\mathrm{G}^{*}\right)^{b} /$ Hartree & -115.6719611 & -115.1789314 & \\
\hline$G_{\text {corr,gas }}^{o}\left(\mathrm{M} 06-2 \mathrm{X} / 6-31+\mathrm{G}^{*}\right)^{c} / \mathrm{kJ} \mathrm{mol}^{-1}$ & 76.7 & 39.1 & -26.3 \\
\hline$G_{c o r r, a q}^{o}\left(\mathrm{M} 06-2 \mathrm{X} / 6-31+\mathrm{G}^{*}\right)^{c} / \mathrm{kJ} \mathrm{mol}^{-1}$ & 76.5 & 46.0 & \\
\hline$\Delta G_{S}^{*} / \mathrm{kJ} / \mathrm{mol}$ & -24.0 & -342.4 & -1112.5 \\
\hline$\Delta G_{g a s}^{o} / \mathrm{kJ} \mathrm{mol}^{-1}$ & 1577.9 & & \\
\hline$\Delta \Delta G_{S}^{*} / \mathrm{kJ} \mathrm{mol}^{-1}$ & -1430.9 & & \\
\hline$\Delta G_{a q}^{*} / \mathrm{kJ} \mathrm{mol}^{-1}$ & 155.0 & & \\
\hline $\mathrm{p} K \mathrm{a}$ & 27.2 & & \\
\hline $\mathrm{p} K \mathrm{a}($ Expt $)$ & 15.5 & & \\
\hline
\end{tabular}

Table S1. Computational data for TC approach ${ }^{a}$

${ }^{a}$ Geometries and thermal corrections evaluated at the M06-2X/6-31+G(d) level of theory.

${ }^{b}$ Includes the non-electrostatic contribution $\left(G_{\text {nes }}\right)$.

${ }^{c}$ Calculated using the rigid-rotor quasi-harmonic oscillator approximation - see text for details. 
Explanatory notes. The gas phase and solution phase electronic energies and thermal corrections are evaluated on geometries optimized in the respective phases. The nonelectrostatic component $\left(G_{\text {nes }}\right)$ is included in the solution phase electronic energy $\left(E_{\text {soln }}\right)$. The thermal corrections were computed using the rigid-rotor quasiharmonic oscillator (RR-QHO) approximation and includes translational, rotational and vibrational contributions. As noted in Refs 9 and 12, the (unhindered) rotational contribution to the reaction energy is generally very small and should not introduce significant errors. Geometries and thermal corrections were evaluated at the M06-2X/6-31+G(d) level of theory, and high-level corrections $(\mathrm{H}=$ G3(MP2)-RAD(+)) were carried out as single-point calculations on the optimized geometries in the gas phase. 
S2. Worked example: Procedure for calculating the $\mathrm{pKa}$ of methanol (at $298 \mathrm{~K}$ ) via the direct approach and the SMD-M062X solvation model.

Direct calculation of solution phase dissociation free energies:

$$
\begin{aligned}
& \Delta G_{\mathrm{soln}}^{*}=\Delta E_{\mathrm{soln}}^{\mathrm{H}}+\Delta G_{\mathrm{corr}}^{\mathrm{soln}, \mathrm{L}}+G_{\mathrm{soln}}^{*}\left(\mathrm{H}^{+}\right) \\
& \Delta E_{\mathrm{soln}}^{H}=E_{\mathrm{soln}}^{H}\left(A^{-}\right)-E_{\mathrm{soln}}^{H}(H A)
\end{aligned}
$$

The high-level single-point calculations and thermal corrections are computed directly within the SMD solvation model on the solution-phase optimized geometry. The non-electrostatic contribution $\left(G_{\text {nes }}\right)$ is included in the solution phase electronic energy $\left(E_{\text {soln }}\right)$.

\begin{tabular}{|c|c|c|c|}
\hline & Methanol & Methoxide & $\mathrm{H}^{+}$ \\
\hline $\mathrm{E}_{\mathrm{aq}}\left[\mathrm{ROCCSD}(\mathrm{T}) / 6-31+\mathrm{G}^{*}\right]^{b} /$ Hartree & -115.3957611 & -114.9029469 & \\
\hline $\mathrm{E}_{\mathrm{aq}}[\mathrm{ROMP}$ /GTMP2Large / Hartree & -115.5153155 & -115.0170003 & \\
\hline $\mathrm{E}_{\mathrm{aq}}\left[\mathrm{ROMP} 2 / 6-31+\mathrm{G}^{*}\right] /$ Hartree & -115.3675704 & -114.8789231 & \\
\hline Higher-level correction / Hartree & -0.06589100 & -0.06589100 & \\
\hline $\mathrm{E}_{\mathrm{aq}}[\mathrm{G} 3(\mathrm{MP} 2)-\mathrm{RAD}(+)]^{b} /$ Hartree & -115.6093972 & -115.1069151 & \\
\hline$G_{c o r r, a q}^{o}(\mathrm{M} 06-2 \mathrm{X})^{c} / \mathrm{kJ} \mathrm{mol}^{-1}$ & 76.5 & 46.0 & \\
\hline$G_{a q}^{*} / \mathrm{kJ} \mathrm{mol}^{-1}$ & -3032823.0 & -301994.2 & -1130.9 \\
\hline$\Delta G_{a q}^{*} / \mathrm{kJ} \mathrm{mol}^{-1}$ & 157.9 & & \\
\hline $\mathrm{p} K \mathrm{a}$ & 27.7 & & \\
\hline $\mathrm{p} K \mathrm{a}(\mathrm{Expt})$ & 15.5 & & \\
\hline
\end{tabular}

Table S2. Computational data for direct approach ${ }^{a}$

${ }^{a}$ Geometries and thermal corrections evaluated at the M06-2X/6-31+G(d) level of theory.

${ }^{b}$ Includes the non-electrostatic contribution $\left(G_{\text {nes }}\right)$.

${ }^{c}$ Calculated using the quasi-harmonic oscillator approximation - see text for details. 
Table S3. Aqueous $\mathrm{p} K \mathrm{a}$ values calculated via a thermodynamic cycle and directly within the continuum solvation model.

\begin{tabular}{|c|c|c|c|c|c|c|c|c|c|c|}
\hline \multirow[b]{2}{*}{ Acid } & \multirow[b]{2}{*}{ Alcohols } & \multicolumn{2}{|c|}{ SMD-M062X $^{c}$} & \multicolumn{2}{|c|}{ SMD-HF $^{d}$} & \multicolumn{2}{|c|}{ CPCM-UAKS $^{e}$} & \multicolumn{2}{|c|}{ CPCM-UAHF $^{d}$} & \multirow[b]{2}{*}{ Expt $\mathrm{p} K \mathrm{a}^{f}$} \\
\hline & & $\mathrm{pKa}(\mathrm{D})^{a}$ & $\mathrm{pKa}(\mathrm{TC})^{b}$ & $\mathrm{pKa}(\mathrm{D})^{a}$ & $\mathrm{pKa}(\mathrm{TC})^{b}$ & $\mathrm{pKa}(\mathrm{D})^{a}$ & $\mathrm{pKa}(\mathrm{TC})^{b}$ & $\mathrm{pKa}(\mathrm{D})^{a}$ & $\mathrm{pKa}(\mathrm{TC})^{b}$ & \\
\hline 1 & $\mathrm{CH}_{3} \mathrm{OH}$ & 27.7 & 27.2 & 26.9 & 24.4 & 26.5 & 26.9 & 29.9 & 26.5 & 15.5 \\
\hline 2 & $\mathrm{CH}_{3} \mathrm{CH}_{2} \mathrm{OH}$ & 27.3 & 26.5 & 26.5 & 23.3 & 26.1 & 26.5 & 29.4 & 25.1 & 15.9 \\
\hline 4 & $\mathrm{C}\left(\mathrm{CH}_{3}\right)_{3} \mathrm{OH}$ & 27.8 & 27.0 & 27.1 & 23.9 & 26.9 & 27.4 & 30.2 & 26.2 & 18.0 \\
\hline 5 & $\mathrm{C}_{6} \mathrm{H}_{5} \mathrm{CH}_{2} \mathrm{OH}$ & 25.3 & 24.7 & 24.7 & 22.0 & 22.1 & 23.0 & 25.9 & 22.3 & 15.4 \\
\hline 6 & $\mathrm{CH}_{3} \mathrm{CH}(\mathrm{OH}) \mathrm{CH}_{3}$ & 27.3 & 26.5 & 26.6 & 23.3 & 26.3 & 26.7 & 29.5 & 25.3 & 17.1 \\
\hline 8 & $\mathrm{C}_{6} \mathrm{H}_{5} \mathrm{OH}$ & 16.3 & 16.3 & 16.0 & 14.2 & 16.5 & 17.0 & 16.6 & 14.1 & 10.0 \\
\hline 9 & p-F- $\mathrm{C}_{6} \mathrm{H}_{4} \mathrm{OH}$ & 16.2 & 16.4 & 16.2 & 14.5 & 16.2 & 17.0 & 19.5 & 17.1 & 10.0 \\
\hline 10 & p- $\mathrm{NH}_{2}-\mathrm{C}_{6} \mathrm{H}_{4} \mathrm{OH}$ & 17.7 & 17.7 & 17.4 & 16.5 & 18.1 & 18.3 & 23.0 & 20.2 & 10.3 \\
\hline 11 & $\mathrm{p}-\mathrm{NO}_{2}-\mathrm{C}_{6} \mathrm{H}_{4} \mathrm{OH}$ & 10.5 & 11.1 & 10.0 & 9.6 & 10.9 & 12.1 & 14.2 & 13.4 & 7.2 \\
\hline 12 & p-Me- $\mathrm{C}_{6} \mathrm{H}_{4} \mathrm{OH}$ & 16.8 & 16.8 & 16.5 & 14.3 & 17.1 & 17.5 & 20.4 & 17.6 & 10.1 \\
\hline 16 & $\mathrm{CF}_{3} \mathrm{COOH}$ & -0.3 & 0.0 & -0.8 & -1.6 & 0.9 & 2.0 & 3.8 & 2.8 & 0.5 \\
\hline 17 & $\mathrm{CCl}_{3} \mathrm{COOH}$ & -0.2 & 0.1 & -0.6 & -1.9 & 1.5 & 2.7 & 4.3 & 2.9 & 0.7 \\
\hline 18 & $\mathrm{CH}_{2} \mathrm{CHCOOH}$ & 7.5 & 7.2 & 7.0 & 5.1 & 8.9 & 9.2 & 11.7 & 9.4 & 4.3 \\
\hline 19 & $\mathrm{C}_{6} \mathrm{H}_{5} \mathrm{COOH}$ & 7.3 & 6.9 & 6.8 & 4.6 & 8.7 & 8.7 & 11.4 & 8.9 & 4.2 \\
\hline \multirow[t]{2}{*}{20} & $\mathrm{CH}_{3} \mathrm{COCOOH}$ & 4.3 & 3.5 & 4.1 & 2.7 & 5.3 & 6.0 & 8.0 & 6.6 & 2.5 \\
\hline & Inorganic acids & & & & & & & & & \\
\hline 21 & HF & 15.2 & 16.0 & 15.1 & 15.2 & 8.5 & 10.6 & 11.9 & 10.8 & 3.2 \\
\hline
\end{tabular}




\begin{tabular}{|c|c|c|c|c|c|c|c|c|c|c|}
\hline 22 & $\mathbf{H C l}$ & 0.0 & 0.7 & -0.2 & 0.0 & -6.1 & -5.1 & -2.8 & -3.1 & -8.0 \\
\hline 23 & $\mathrm{H}_{2} \mathrm{O}$ & 26.3 & 25.4 & & & & & & & 14.0 \\
\hline 24 & $\mathbf{H O O H}$ & 19.5 & 18.9 & 18.6 & 17.2 & 21.7 & 22.1 & 26.8 & 25.9 & 11.7 \\
\hline 25 & $\mathrm{NH}_{3}$ & 43.3 & 41.9 & & & & & & & 33.0 \\
\hline 26 & $\mathrm{H}_{2} \mathrm{~S}$ & 14.3 & 14.5 & 13.8 & 13.6 & 8.5 & 9.1 & 11.8 & 11.0 & 7.0 \\
\hline 27 & $\mathrm{HN}_{3}$ & 6.7 & 7.2 & 6.4 & 6.8 & 8.4 & 9.3 & 14.2 & 14.5 & 4.7 \\
\hline 28 & $\mathrm{HOCl}$ & 13.6 & 12.9 & 12.9 & 12.4 & 14.7 & 14.7 & 18.8 & 18.6 & 7.5 \\
\hline 29 & $\mathrm{HNO}_{2}$ & 3.6 & 3.9 & 2.8 & 2.0 & 7.7 & 8.7 & 11.6 & 11.7 & 3.3 \\
\hline 30 & $\mathrm{HNO}_{3}$ & -4.2 & -3.5 & -5.0 & -4.5 & -1.6 & -0.6 & 2.5 & 3.2 & -1.3 \\
\hline 31 & $\mathbf{H}_{2} \mathrm{SO}_{4}$ & -9.7 & -9.4 & -10.0 & -10.4 & -7.6 & -6.6 & -3.1 & -3.0 & -10.0 \\
\hline 32 & $\mathbf{H}_{3} \mathrm{PO}_{4}$ & 1.0 & 1.6 & 1.1 & 0.2 & 3.4 & 4.5 & 8.4 & 8.1 & 2.1 \\
\hline 33 & $\mathrm{CH}_{3} \mathrm{SO}_{3} \mathbf{H}$ & -5.4 & -5.7 & -6.0 & -7.0 & -3.9 & -3.9 & 0.7 & 0.1 & -2.6 \\
\hline \multirow[t]{2}{*}{34} & $\mathrm{CF}_{3} \mathrm{SO}_{3} \mathbf{H}$ & -14.7 & -14.5 & -15.3 & -15.4 & -11.8 & -11.1 & -7.5 & -7.2 & -14.0 \\
\hline & Carbon acids & & & & & & & & & \\
\hline 35 & $\mathrm{CH}_{3} \mathrm{COCH}_{3}$ & 27.4 & 27.1 & 27.0 & 25.9 & 29.3 & 30.1 & 33.8 & 33.0 & 19 \\
\hline 36 & $\mathrm{CH}_{3} \mathrm{COCH}_{2} \mathrm{COCH}_{3}$ & 14.1 & 13.5 & 13.0 & 11.9 & 15.2 & 15.4 & 20.4 & 19.8 & 9 \\
\hline 37 & $\mathbf{H C C H}$ & 27.5 & 28.2 & 26.9 & 27.0 & 24.9 & 26.3 & 27.9 & 27.8 & 21.7 \\
\hline 38 & $\mathbf{H C N}$ & 16.6 & 16.9 & 16.3 & 16.7 & 15.3 & 16.2 & 15.5 & 14.8 & 9.4 \\
\hline 39 & $\mathrm{CH}_{3} \mathrm{CN}$ & 34.9 & 34.6 & 34.7 & 34.4 & 33.1 & 33.1 & 36.7 & 36.3 & 28.9 \\
\hline 40 & $\mathrm{CH}_{3} \mathrm{CH}_{2} \mathrm{CN}$ & 37.6 & 37.2 & 37.3 & 36.9 & 35.6 & 35.6 & 39.4 & 39.0 & 30.9 \\
\hline 41 & $\mathrm{CH}_{2}(\mathrm{CN})_{2}$ & 15.7 & 15.6 & 14.9 & 15.6 & 12.6 & 12.7 & 16.1 & 16.2 & 11.81 \\
\hline 42 & $\mathrm{CH}_{3} \mathrm{NO}_{2}$ & 17.2 & 17.3 & 16.8 & 15.2 & 18.9 & 20.0 & 24.0 & 23.2 & 10.3 \\
\hline 43 & $\mathrm{CH}_{3} \mathrm{CONH}_{2}$ & 34.3 & 33.6 & 34.0 & 32.8 & 34.6 & 35.0 & 39.1 & 38.2 & 28.4 \\
\hline 44 & $\mathrm{CH}_{3} \mathrm{CON}\left(\mathrm{CH}_{3}\right)_{2}$ & 34.8 & 34.2 & 34.3 & 32.7 & 37.4 & 37.9 & 41.7 & 40.6 & 29.4 \\
\hline 45 & $\mathrm{CH}_{3} \mathrm{CONHCH}_{2} \mathrm{CONH}_{2}$ & 34.4 & 34.1 & 33.8 & 32.5 & 34.9 & 35.4 & 40.4 & 40.5 & 29.1 \\
\hline 46 & $\mathrm{CH}_{3} \mathrm{CONHCH}_{2} \mathrm{CONH}_{2}$ & 31.1 & 30.5 & 31.5 & 28.9 & 31.9 & 32.1 & 38.5 & 37.5 & 23.9 \\
\hline
\end{tabular}




\begin{tabular}{|c|c|c|c|c|c|c|c|c|c|c|}
\hline 47 & $\mathrm{CH}_{3} \mathrm{COOCH}_{2} \mathrm{CH}_{3}$ & 31.7 & 31.1 & 31.2 & 29.8 & 34.1 & 34.6 & 38.4 & 37.5 & 25.6 \\
\hline 48 & & 26.0 & 25.5 & 25.6 & 23.9 & 25.7 & 26.5 & 28.8 & 26.3 & 18.7 \\
\hline 50 & & 37.4 & 37.5 & 36.9 & 37.0 & 37.5 & 38.2 & 41.1 & 41.2 & 30 \\
\hline 52 & & 40.1 & 40.2 & 39.5 & 39.3 & 40.2 & 40.9 & 43.8 & 43.6 & 33 \\
\hline 53 & & 40.8 & 41.0 & 40.3 & 40.2 & 40.5 & 41.3 & 44.1 & 44.1 & 32 \\
\hline & Cationic acids & & & & & & & & & \\
\hline 56 & $+\mathrm{NH}_{2}-\mathrm{CH}_{2}-\mathrm{COOMe}$ & 24.7 & 24.2 & 29.8 & 29.1 & 25.5 & 25.2 & 25.7 & 25.2 & 21 \\
\hline 57 & $+\mathrm{NMe}_{3}-\mathrm{CH}_{2}-\mathrm{COOMe}$ & 24.4 & 24.2 & 24.3 & 24.0 & 24.3 & 24.1 & 24.3 & 24.2 & 18 \\
\hline 58 & $\mathrm{Me}_{2} \mathrm{C}=\mathrm{NH}-\mathrm{CH}_{2}-\mathrm{COOMe}$ & 18.1 & 17.3 & 18.4 & 15.1 & 20.4 & 19.7 & 21.2 & 18.5 & 14 \\
\hline 59 & & 26.1 & 25.1 & 25.6 & 24.7 & 25.7 & 25.3 & 25.9 & 25.4 & 21 \\
\hline
\end{tabular}




\begin{tabular}{|c|c|c|c|c|c|c|c|c|c|c|}
\hline 60 & $\mathrm{H}$ & 25.8 & 26.0 & 25.3 & 25.9 & 24.9 & 24.9 & 21.2 & 21.8 & 23.8 \\
\hline 61 & & 27.1 & 27.2 & 26.6 & 27.3 & 24.4 & 24.5 & 23.9 & 24.3 & 23 \\
\hline 62 & & 24.7 & 24.9 & 24.1 & 25.4 & 21.9 & 22.0 & 21.3 & 22.1 & 21.6 \\
\hline 64 & & 33.9 & 33.9 & 33.7 & 33.8 & 31.3 & 31.3 & 31.0 & 31.1 & 34 \\
\hline 65 & & 33.4 & 33.4 & 33.0 & 33.3 & 31.4 & 31.3 & 31.2 & 31.4 & 33 \\
\hline 68 & $\mathbf{H}+\operatorname{ch} 3 \mathrm{nh} 2$ & 10.1 & 9.5 & 9.7 & 9.2 & 7.5 & 7.1 & 7.2 & 6.6 & 10.67 \\
\hline 69 & $\mathbf{H}+$ allyamine & 8.9 & 8.3 & 8.4 & 7.9 & 7.1 & 6.6 & 6.7 & 6.3 & 9.49 \\
\hline 70 & $\mathbf{H}+$ cyclohexamine & 10.7 & 10.3 & 9.9 & 10.0 & 7.7 & 7.6 & 7.3 & 7.3 & 10.49 \\
\hline 71 & $\mathbf{H}+$ pyrrolidine & 10.7 & 10.4 & 10.1 & 10.7 & 10.3 & 10.2 & 9.9 & 10.0 & 11.27 \\
\hline 72 & $\mathbf{H}+$ dimethylamine & 10.4 & 10.3 & 9.9 & 9.9 & 9.3 & 9.2 & 9.0 & 8.6 & 10.77 \\
\hline 73 & $\mathbf{H}+$ methylsulfonylaniline & -1.8 & -2.0 & -2.3 & -1.4 & -5.2 & -5.6 & -4.6 & -3.8 & 1.48 \\
\hline 74 & $\mathbf{H}+$ aniline & 1.8 & 1.3 & 1.2 & 1.0 & -0.8 & -1.1 & -0.3 & -0.5 & 4.61 \\
\hline 75 & $\mathbf{H}+$ guanidine & 16.4 & 15.6 & 16.1 & 14.7 & 17.8 & 16.9 & 16.7 & 15.3 & 13.6 \\
\hline 76 & $\mathbf{H}+$ acetamidine & 14.0 & 13.2 & 13.8 & 12.6 & 13.7 & 13.0 & 13.5 & 12.1 & 12.1 \\
\hline 77 & $\mathbf{H}+$ pyridine & 4.2 & 4.1 & 3.8 & 3.7 & 3.6 & 3.4 & 1.9 & 1.6 & 5.25 \\
\hline
\end{tabular}




\begin{tabular}{|c|c|c|c|c|c|c|c|c|c|c|}
\hline 78 & $\mathbf{H}+$ aminopyridine & 8.6 & 8.5 & 8.5 & 8.3 & 9.1 & 8.6 & 7.3 & 6.9 & 9.18 \\
\hline 79 & $\mathbf{H}+$ pyrazine & -1.4 & -1.2 & -1.6 & -1.4 & 0.0 & 0.0 & -1.9 & -2.0 & 1.1 \\
\hline 80 & $\mathbf{H}+$ isoquinoline & 4.2 & 4.2 & 3.9 & 4.1 & 4.2 & 3.7 & 2.6 & 2.4 & 5.38 \\
\hline 81 & $\mathbf{H}+$ pyrimidine & -0.3 & -0.3 & -0.6 & -0.5 & 0.0 & -0.1 & -1.9 & -2.1 & -7.7 \\
\hline 82 & $\mathbf{H}+$ morpholine & 8.7 & 8.6 & 8.1 & 8.3 & 9.3 & 9.4 & 8.9 & 9.0 & 8.49 \\
\hline 83 & $\mathbf{H}+$ benzothiazole & -2.0 & -1.8 & -2.1 & -0.9 & -0.1 & -0.2 & -2.0 & -1.4 & 1.2 \\
\hline
\end{tabular}

${ }^{a}$ Aqueous $\mathrm{pKa}$ values calculated directly within the continuum solvation model via eqn (S2).

${ }^{b}$ Aqueous $\mathrm{pKa}$ values calculated via the thermodynamic cycle using eqn (S1).

${ }^{c}$ Geometries and thermal corrections calculated at the M06-2X/6-31+G(d) level of theory in the respective phases.

${ }^{d}$ Geometries and thermal corrections calculated at the HF/6-31+G(d) level of theory in the respective phases.

${ }^{e}$ Geometries and thermal corrections calculated at the B3LYP/6-31+G(d) level of theory in the respective phases.

${ }^{f}$ See ref 4 a for details. 
Table S4 Aqueous reduction potentials (in eV) calculated via a thermodynamic cycle and directly within the various continuum solvation models.

\begin{tabular}{|c|c|c|c|c|c|c|c|c|c|c|c|}
\hline \multicolumn{3}{|c|}{ Amines } & \multicolumn{2}{|c|}{ SMD-M062X ${ }^{c}$} & \multicolumn{2}{|c|}{ SMD-HF $^{d}$} & \multicolumn{2}{|c|}{ CPCM-UAKS ${ }^{e}$} & \multicolumn{2}{|c|}{$\mathbf{C P C M}$-UAHF $^{d}$} & \multirow[b]{2}{*}{$\operatorname{Expt~E}^{\circ}$} \\
\hline & $\mathrm{Ox}$ & Red & $\mathrm{E}^{\mathrm{o}}(\mathrm{D})^{a}$ & $\mathrm{E}^{\mathrm{o}}(\mathrm{TC})^{b}$ & $\mathrm{E}^{\mathrm{o}}(\mathrm{D})^{a}$ & $\mathrm{E}^{\mathrm{o}}(\mathrm{TC})^{b}$ & $\mathrm{E}^{\mathrm{o}}(\mathrm{D})^{a}$ & $\mathrm{E}^{\mathrm{o}}(\mathrm{TC})^{b}$ & $\mathrm{E}^{\mathrm{o}}(\mathrm{D})^{a}$ & $\mathrm{E}^{\mathrm{o}}(\mathrm{TC})^{b}$ & \\
\hline 1 & $\mathrm{Me}_{2} \mathrm{NH}^{+}$ & $\mathrm{Me}_{2} \mathrm{NH}$ & 1.24 & 1.25 & 1.27 & 1.25 & 1.25 & 1.26 & 1.07 & 1.07 & 1.27 \\
\hline 2 & $\mathrm{Et}_{2} \mathrm{NH}^{\cdot+}$ & $\mathrm{Et}_{2} \mathrm{NH}$ & 1.22 & 1.23 & 1.26 & 1.22 & 1.25 & 1.27 & 1.08 & 1.07 & 1.36 \\
\hline 3 & Piperidine $^{.+}$ & Piperidine & 1.25 & 1.26 & 1.30 & 1.26 & 1.22 & 1.25 & 1.05 & 1.03 & 1.34 \\
\hline 4 & $\mathrm{PhNH}_{2}^{+}$ & PhNH2 & 1.12 & 1.16 & 1.07 & 1.16 & 1.22 & 1.25 & 1.05 & 1.13 & 1.02 \\
\hline 5 & $4 \mathrm{OH}-\mathrm{PhNH}_{2}^{.+}$ & $4 \mathrm{OH}-\mathrm{PhNH}_{2}$ & 0.83 & 0.87 & 0.78 & 0.85 & 0.84 & 0.86 & 0.67 & 0.73 & 0.76 \\
\hline 6 & $4 \mathrm{NH}_{2}-\mathrm{Ph}-\mathrm{NH}_{2}{ }^{+}$ & $4 \mathrm{NH}_{2}-\mathrm{Ph}-\mathrm{NH}_{2}$ & 0.43 & 0.47 & 0.32 & 0.37 & 0.45 & 0.48 & 0.30 & 0.35 & 0.59 \\
\hline 7 & $4 \mathrm{Me}-\mathrm{PhNH}_{2}{ }^{+}$ & $4 \mathrm{Me}-\mathrm{PhNH}_{2}$ & 0.98 & 1.01 & 0.94 & 1.02 & 1.08 & 1.10 & 0.91 & 0.99 & 0.92 \\
\hline 8 & $4 \mathrm{MeO}-\mathrm{PhNH}_{2}^{.+}$ & $4 \mathrm{MeO}-\mathrm{PhNH}_{2}$ & 0.78 & 0.82 & 0.73 & 0.80 & 0.86 & 0.89 & 0.67 & 0.74 & 0.79 \\
\hline 9 & $4 \mathrm{COCH}_{3}-\mathrm{PhNH}_{2}{ }^{+}$ & $4 \mathrm{COCH}_{3}-\mathrm{PhNH}_{2}$ & 1.35 & 1.40 & 1.30 & 1.38 & 1.47 & 1.53 & 1.28 & 1.36 & 1.14 \\
\hline 10 & $4 \mathrm{CN}-\mathrm{PhNH}_{2}^{.+}$ & $4 \mathrm{CN}-\mathrm{PhNH}_{2}$ & 1.42 & 1.49 & 1.34 & 1.40 & 1.60 & 1.70 & 1.38 & 1.46 & 1.32 \\
\hline 11 & $\mathrm{Ph}_{2} \mathrm{NH}^{+}$ & $\mathrm{Ph}_{2} \mathrm{NH}$ & 0.98 & 1.01 & 0.86 & 0.85 & 1.09 & 1.13 & 0.84 & 0.83 & 1.0 \\
\hline 12 & Indole $^{+}$ & Indole & 1.33 & 1.35 & 1.28 & 1.23 & 1.46 & 1.48 & 1.25 & 1.22 & 1.24 \\
\hline 13 & 3-methyl-indole ${ }^{++}$ & 3-methyl-indole & 1.15 & 1.17 & 1.10 & 1.04 & 1.28 & 1.30 & 1.07 & 1.06 & 1.07 \\
\hline \multirow[t]{2}{*}{14} & 2,3-dimethyl-indole ${ }^{+}$ & 2,3-dimethyl-indole & 0.94 & 0.95 & 0.90 & 0.84 & 1.08 & 1.09 & 0.87 & 0.84 & 0.93 \\
\hline & Nitroxides & & $\mathrm{E}^{\mathrm{o}}(\mathrm{D})^{a}$ & $\mathrm{E}^{\mathrm{o}}(\mathrm{TC})^{b}$ & $\mathrm{E}^{\mathrm{o}}(\mathrm{D})^{a}$ & $\mathrm{E}^{\mathrm{o}}(\mathrm{TC})^{b}$ & $\mathrm{E}^{\mathrm{o}}(\mathrm{D})^{a}$ & $\mathrm{E}^{\mathrm{o}}(\mathrm{TC})^{b}$ & $\mathrm{E}^{\mathrm{o}}(\mathrm{D})^{a}$ & $\mathrm{E}^{\mathrm{o}}(\mathrm{TC})^{b}$ & $\operatorname{Expt~}^{o}$ \\
\hline 15 & 0 & o. & 0.55 & 0.60 & 0.65 & 0.68 & 0.76 & 0.77 & 0.62 & 0.63 & 0.74 \\
\hline
\end{tabular}


16<smiles>CC1(C)CC(O)CC(C)(C)[NH2+]1</smiles>

17

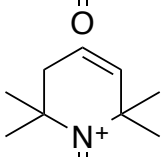

$\mathrm{O}$

18<smiles>CC1(C)C=C(C(N)=O)C(C)(C)[N+]1=O</smiles><smiles>CC1(C)CC(O)CC(C)(C)N1</smiles>

O.<smiles>CC1(C)C=CCC(C)(C)N1</smiles>

O.<smiles>CC1(C)C=C(C(N)=O)C(C)(C)N1</smiles>

O.
0.70

0.75

0.73

0.76

0.78

0.79

0.63

0.64

0.825

0.68

0.72

0.71

0.74

0.82

0.83

0.67

0.68

0.795

$\mathrm{CONH}_{2}$

0.81

0.87

0.85

0.86

1.00

1.02

0.85

0.84

0.955

\begin{tabular}{|c|c|c|c|c|c|c|c|c|c|c|c|}
\hline & Alcohols & & $\mathrm{E}^{\mathrm{o}}(\mathrm{D})^{a}$ & $\mathrm{E}^{\mathrm{o}}(\mathrm{TC})^{b}$ & $\mathrm{E}^{\mathrm{o}}(\mathrm{D})^{a}$ & $\mathrm{E}^{\mathrm{o}}(\mathrm{TC})^{b}$ & $\mathrm{E}^{\mathrm{o}}(\mathrm{D})^{a}$ & $\mathrm{E}^{\mathrm{o}}(\mathrm{TC})^{b}$ & $\mathrm{E}^{\mathrm{o}}(\mathrm{D})^{a}$ & $\mathrm{E}^{\mathrm{o}}(\mathrm{TC})^{b}$ & $\operatorname{Expt~} \mathrm{E}^{\mathrm{o}}$ \\
\hline 19 & $\mathrm{PhO}^{\circ}$ & $\mathrm{PhO}^{-}$ & 0.45 & 0.42 & 0.38 & 0.31 & 0.51 & 0.37 & 0.25 & 0.26 & 0.794 \\
\hline 20 & 4F-PhO & $4 \mathrm{~F}-\mathrm{PhO}^{-}$ & 0.40 & 0.35 & 0.32 & 0.29 & 0.47 & 0.27 & 0.20 & 0.24 & 0.76 \\
\hline 21 & 4CN-PhO• & 4CN-PhO ${ }^{-}$ & 0.80 & 0.80 & 0.71 & 0.64 & 0.93 & 0.90 & 0.63 & 0.64 & 1.12 \\
\hline 22 & $4 \mathrm{COCH}_{3}-\mathrm{PhO}$ & $4 \mathrm{COCH}_{3}-\mathrm{PhO}^{-}$ & 0.76 & 0.75 & 0.69 & 0.58 & 0.83 & 0.79 & 0.56 & 0.52 & 1.0 \\
\hline 23 & $4 \mathrm{NO}_{2}-\mathrm{PhO}$ & $4 \mathrm{NO}_{2}-\mathrm{PhO}^{-}$ & 0.99 & 0.98 & 0.93 & 0.95 & 1.05 & 0.95 & 0.81 & 0.87 & 1.22 \\
\hline 24 & $4 \mathrm{NMe}_{2}-\mathrm{PhO}$ & $4 \mathrm{NMe}_{2}-\mathrm{PhO}^{-}$ & -0.21 & -0.24 & -0.17 & -0.18 & -0.16 & -0.23 & -0.36 & -0.28 & 0.174 \\
\hline 25 & $4 \mathrm{MeO}-\mathrm{PhO}$ & $4 \mathrm{MeO}-\mathrm{PhO}^{-}$ & 0.14 & 0.12 & 0.07 & 0.05 & 0.21 & 0.15 & -0.06 & 0.01 & 0.54 \\
\hline 26 & 4OH-PhO• & $4 \mathrm{OH}-\mathrm{PhO}^{-}$ & 0.16 & 0.14 & 0.09 & 0.01 & 0.19 & 0.11 & -0.07 & -0.06 & 0.45 \\
\hline 27 & 1-naphthol' & 1-naphthol ${ }^{-}$ & 0.24 & 0.21 & 0.12 & 0.08 & 0.32 & 0.26 & 0.00 & 0.04 & 0.59 \\
\hline 28 & $\mathrm{PhOH}^{+}$ & $\mathrm{PhOH}$ & 0.26 & 0.26 & 0.18 & 0.28 & 0.53 & 0.48 & 0.27 & 0.42 & 1.5 \\
\hline 29 & $4 \mathrm{MeO}^{-\mathrm{PhOH}^{+}}$ & $4 \mathrm{MeO}-\mathrm{PhOH}$ & 0.08 & 0.07 & 0.01 & 0.12 & 0.38 & 0.32 & 0.11 & 0.28 & 1.1 \\
\hline 30 & $\mathrm{OH}-\mathrm{PhOH}^{+}$ & $\mathrm{OH}-\mathrm{Ph}-\mathrm{OH}$ & -0.09 & -0.12 & -0.17 & 0.00 & 0.18 & 0.11 & -0.02 & 0.18 & 1.1 \\
\hline 31 & $\mathrm{PhS}$ & $\mathrm{PhS}^{-}$ & 0.67 & 0.67 & 0.67 & 0.70 & 1.00 & 0.98 & 0.81 & 0.86 & 0.69 \\
\hline 32 & 4MeO-PhS & $4 \mathrm{MeO}-\mathrm{PhS}^{-}$ & 0.05 & 0.06 & 0.07 & 0.12 & 0.44 & 0.44 & 0.26 & 0.37 & 0.57 \\
\hline 33 & $4 \mathrm{NH}_{2}-\mathrm{PhS}$ & $4 \mathrm{NH}_{2}-\mathrm{PhS}^{-}$ & 0.88 & 0.84 & 0.81 & 0.90 & 0.68 & 0.64 & 0.40 & 0.47 & 0.36 \\
\hline
\end{tabular}




\begin{tabular}{|c|c|c|c|c|c|c|c|c|c|c|c|}
\hline 34 & $\mathrm{HS}^{-}$ & $\mathrm{HS}^{-}$ & 0.97 & 0.99 & 0.98 & 1.05 & 1.03 & 1.02 & 0.76 & 0.84 & 1.15 \\
\hline 35 & $\mathrm{CH}_{3} \mathrm{~S}^{\cdot}$ & $\mathrm{CH}_{3} \mathrm{~S}^{-}$ & 0.96 & 0.97 & 0.96 & 1.03 & 1.01 & 1.00 & 0.74 & 0.82 & 0.73 \\
\hline 36 & Ascorbate & Ascorbate $^{-}$ & 0.94 & 0.96 & 0.93 & 1.01 & 1.00 & 0.99 & 0.72 & 0.80 & 0.72 \\
\hline 37 & PhCOS & $\mathrm{PhCOS}^{-}$ & 0.92 & 0.93 & 0.93 & 1.01 & 1.01 & 1.00 & 0.75 & 0.84 & 1.21 \\
\hline 39 & $4 \mathrm{MeOPhCOS}$ & $4 \mathrm{MeoPhCOS}^{-}$ & 0.89 & 0.96 & 0.92 & 1.12 & 0.88 & 0.82 & 0.74 & 0.99 & 1.17 \\
\hline 40 & EtCOS & $\mathrm{EtCOS}^{-}$ & 1.90 & 1.91 & 1.87 & 1.89 & 2.01 & 2.01 & 1.79 & 1.83 & 1.22 \\
\hline 41 & $\mathrm{Me}_{2} \mathrm{COHO}$ & $\mathrm{Me}_{2} \mathrm{COHO}^{-}$ & 1.36 & 1.38 & 1.32 & 1.32 & 1.44 & 1.44 & 1.22 & 1.23 & 1.45 \\
\hline
\end{tabular}

${ }^{a}$ Aqueous reduction potentials calculated directly within the continuum solvation model.

${ }^{b}$ Aqueous reduction potentials calculated via the thermodynamic cycle (Figure 1 cycle B) employing G3(MP2)-RAD $(+)$ single-point gas phase energies with solvation free energies computed from eqn (1).

${ }^{c}$ Geometries and thermal corrections calculated at the M06-2X/6-31+G(d) level of theory in the respective phases.

${ }^{d}$ Geometries and thermal corrections calculated at the HF/6-31+G(d) level of theory in the respective phases.

${ }^{e}$ Geometries and thermal corrections calculated at the B3LYP/6-31+G(d) level of theory in the respective phases.

${ }^{f}$ See ref 5a for details. 
Table S5. Cluster-continuum $\mathrm{p} K$ as calculated in conjunction with the SMD-M062X solvation model.

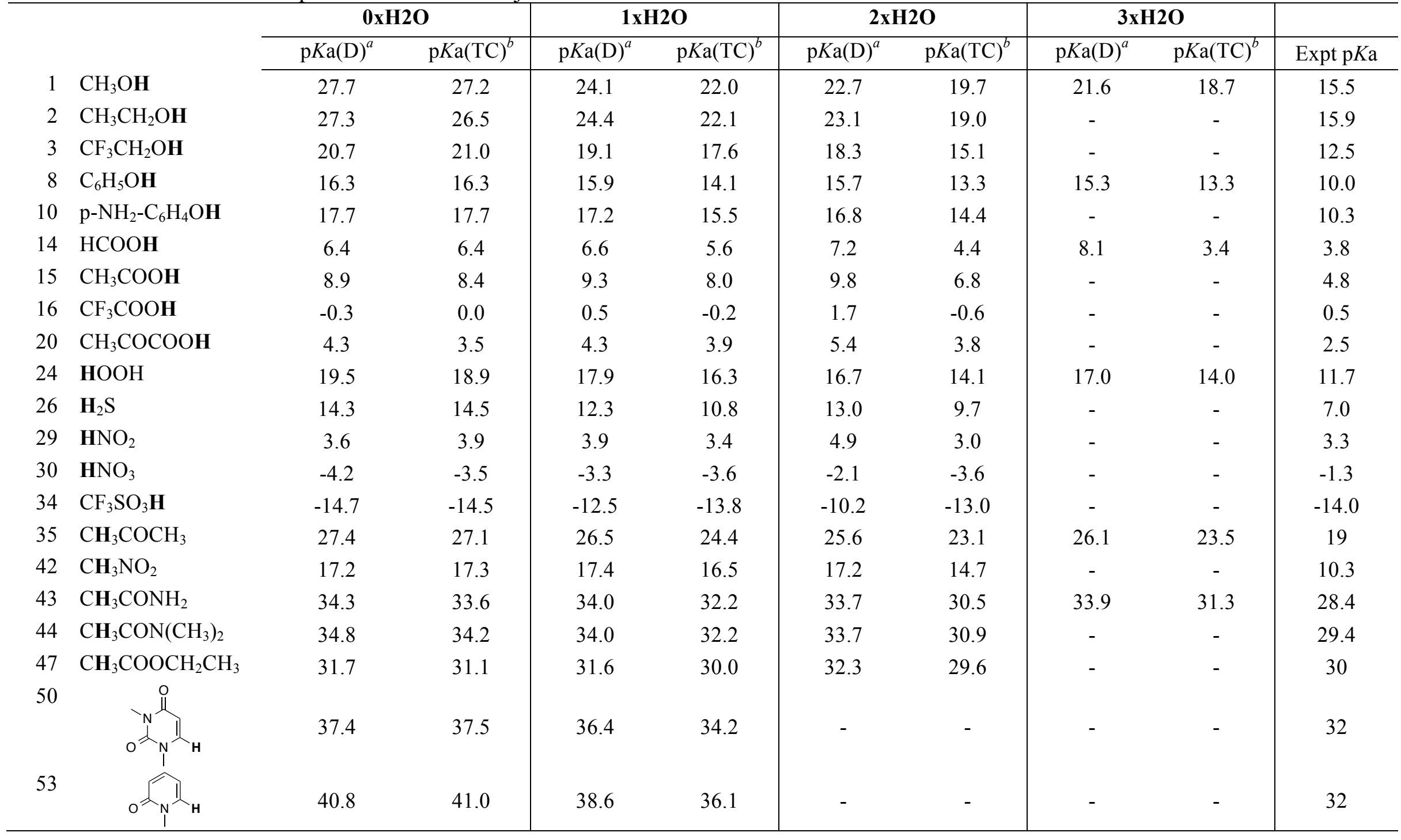




\begin{tabular}{lll|ll|l|l}
\hline 54 & 40.1 & 40.2 & 38.2 & 35.8 & - & - \\
& & & - & -19 \\
\hline
\end{tabular}

${ }^{a}$ Aqueous $\mathrm{pKas}$ calculated directly within the continuum solvation model using eqn (9) in text.

${ }^{b}$ Aqueous $\mathrm{p}$ Kas calculated via the thermodynamic cycle (Figure 1 cycle C) using eqn (8) in text. 
Table S6. Cluster-continuum $\mathrm{pK}$ as calculated in conjunction with the SMD-HF solvation model.

\begin{tabular}{|c|c|c|c|c|c|c|c|c|c|c|}
\hline & \multicolumn{2}{|c|}{$0 \times H 2 O$} & \multicolumn{2}{|c|}{$1 \times H 2 O$} & \multicolumn{2}{|c|}{$2 \times H 2 O$} & \multicolumn{2}{|c|}{$3 \times H 2 O$} & \multirow[b]{2}{*}{ Expt $\mathrm{p} K \mathrm{a}$} \\
\hline & & $\mathrm{p} K \mathrm{a}(\mathrm{D})^{a}$ & $\mathrm{p} K \mathrm{a}(\mathrm{TC})^{b}$ & $\mathrm{p} K \mathrm{a}(\mathrm{D})^{a}$ & $\mathrm{p} K \mathrm{a}(\mathrm{TC})^{b}$ & $\mathrm{p} K \mathrm{a}(\mathrm{D})^{a}$ & $\mathrm{p} K \mathrm{a}(\mathrm{TC})^{b}$ & $\mathrm{p} K \mathrm{a}(\mathrm{D})^{a}$ & $\mathrm{p} K \mathrm{a}(\mathrm{TC})^{b}$ & \\
\hline 1 & $\mathrm{CH}_{3} \mathrm{OH}$ & 26.9 & 24.4 & 24.5 & 21.2 & 22.6 & 18.5 & 21.2 & 16.1 & 15.5 \\
\hline 2 & $\mathrm{CH}_{3} \mathrm{CH}_{2} \mathrm{OH}$ & 26.5 & 23.3 & 24.9 & 21.2 & 23.3 & 19.0 & - & - & 15.9 \\
\hline 3 & $\mathrm{CF}_{3} \mathrm{CH}_{2} \mathrm{OH}$ & 20.4 & 18.9 & 19.8 & 17.4 & 18.9 & 15.6 & - & - & 12.5 \\
\hline 8 & $\mathrm{C}_{6} \mathrm{H}_{5} \mathrm{OH}$ & 16.0 & 14.2 & 16.1 & 13.2 & 15.9 & 12.0 & 15.6 & 10.4 & 10.0 \\
\hline 10 & $\mathrm{p}-\mathrm{NH}_{2}-\mathrm{C}_{6} \mathrm{H}_{4} \mathrm{OH}$ & 17.4 & 16.5 & 17.8 & 15.8 & 16.9 & 14.5 & 16.6 & 12.7 & 10.3 \\
\hline 14 & НСОOH & 5.9 & 4.5 & 6.5 & 3.4 & 6.8 & 2.3 & - & - & 3.8 \\
\hline 15 & $\mathrm{CH}_{3} \mathrm{COOH}$ & 8.3 & 6.3 & 9.2 & 5.7 & 9.5 & 4.7 & - & - & 4.8 \\
\hline 16 & $\mathrm{CF}_{3} \mathrm{COOH}$ & -0.8 & -1.6 & 0.5 & -2.1 & 1.1 & -2.9 & - & - & 0.5 \\
\hline 20 & $\mathrm{CH}_{3} \mathrm{COCOOH}$ & 4.1 & 2.7 & 5.5 & 2.6 & 6.2 & 1.7 & - & - & 2.5 \\
\hline 24 & $\mathbf{H O O H}$ & 18.6 & 17.2 & 17.5 & 14.3 & 16.4 & 11.9 & 16.0 & 10.0 & 11.7 \\
\hline 26 & $\mathbf{H}_{2} \mathrm{~S}$ & 13.8 & 13.6 & 12.1 & 10.3 & 12.4 & 9.3 & 12.0 & 7.0 & 7.0 \\
\hline 29 & $\mathbf{H N O}_{2}$ & 2.8 & 2.0 & 3.3 & 0.8 & 3.7 & -0.6 & - & - & 3.3 \\
\hline 30 & $\mathbf{H N O}_{3}$ & -5.0 & -4.5 & -3.9 & -5.3 & -2.9 & -6.0 & - & - & -1.3 \\
\hline 34 & $\mathrm{CF}_{3} \mathrm{SO}_{3} \mathrm{H}$ & -15.3 & -15.4 & -13.4 & -15.5 & -11.8 & -15.9 & - & - & -14.0 \\
\hline 35 & $\mathrm{CH}_{3} \mathrm{COCH}_{3}$ & 27.0 & 25.9 & 26.8 & 24.7 & 26.1 & 22.8 & 25.8 & 21.0 & 19 \\
\hline 42 & $\mathrm{CH}_{3} \mathrm{NO}_{2}$ & 16.8 & 15.2 & 17.1 & 14.1 & 16.8 & 12.1 & - & - & 10.3 \\
\hline 43 & $\mathrm{CH}_{3} \mathrm{CONH}_{2}$ & 34.0 & 32.8 & 33.9 & 31.5 & 33.7 & 29.9 & 33.6 & 28.5 & 28.4 \\
\hline 44 & $\mathrm{CH}_{3} \mathrm{CON}\left(\mathrm{CH}_{3}\right)_{2}$ & 34.3 & 32.7 & 34.2 & 31.9 & 34.2 & 30.6 & - & - & 29.4 \\
\hline 47 & $\mathrm{CH}_{3} \mathrm{COOCH}_{2} \mathrm{CH}_{3}$ & 31.2 & 29.8 & 31.6 & 28.9 & 31.6 & 27.8 & 31.8 & 26.0 & 30 \\
\hline \multicolumn{11}{|l|}{50} \\
\hline & & 36.9 & 37.0 & 36.9 & 35.2 & - & - & - & - & 32 \\
\hline 53 & & 40.3 & 40.2 & 39.4 & 37.6 & - & - & - & - & 32 \\
\hline
\end{tabular}




\begin{tabular}{lll|ll|l|l}
\hline 54 & 39.7 & 39.0 & 39.3 & 37.0 & - & - \\
& & & - & -19 \\
\hline
\end{tabular}

${ }^{a}$ Aqueous $\mathrm{pKas}$ calculated directly within the continuum solvation model using eqn (9) in text.

${ }^{b}$ Aqueous $\mathrm{p}$ Kas calculated via the thermodynamic cycle (Figure 1 cycle C) using eqn (8) in text. 
Table S7. Cluster-continuum $\mathrm{pKas}$ calculated in conjunction with the CPCM-UAKS solvation model.

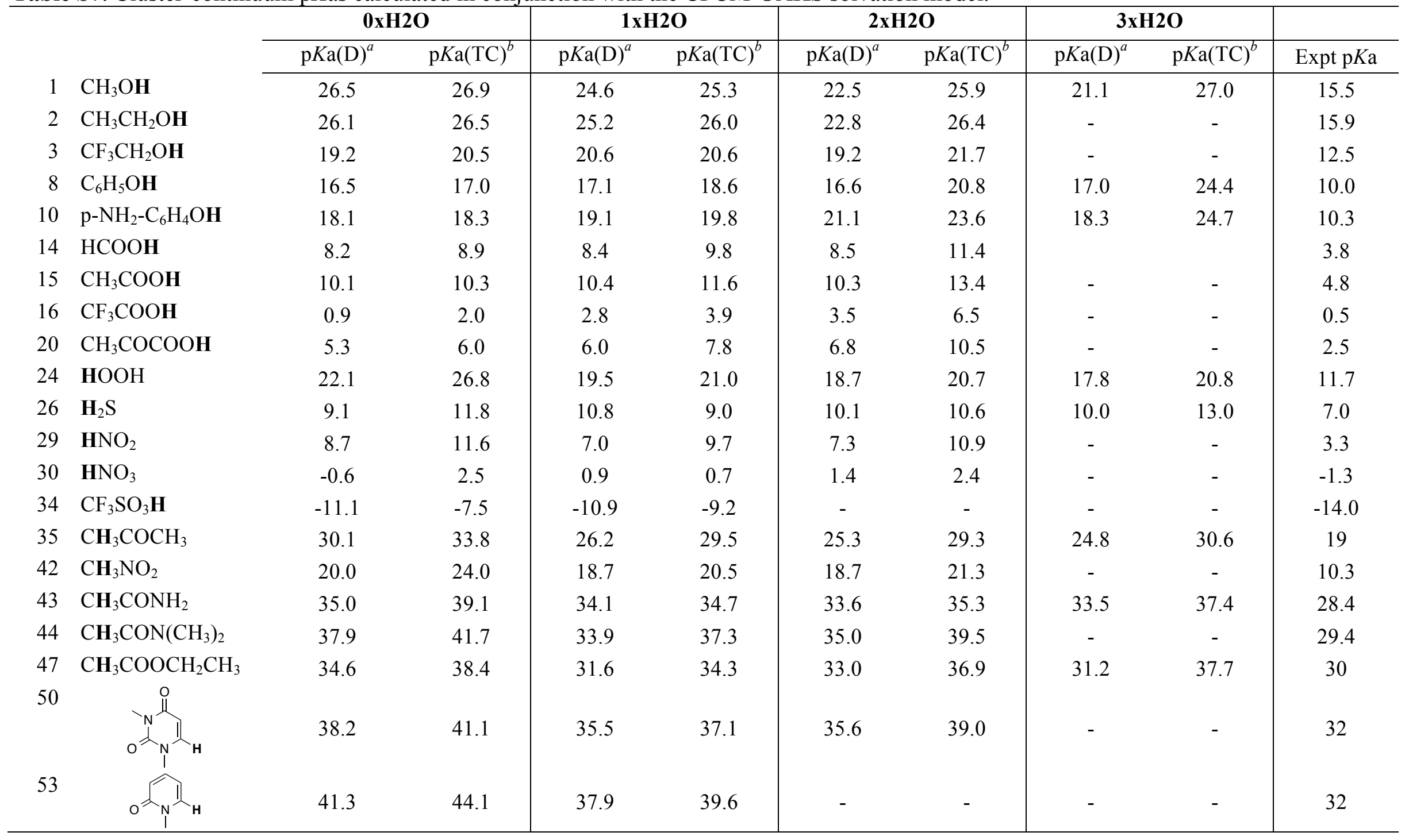




\begin{tabular}{lll|ll|l|l}
\hline 54 & 39.1 & 41.8 & 37.4 & 37.8 & - & - \\
& & & & - \\
\hline
\end{tabular}

${ }^{a}$ Aqueous pKas calculated directly within the continuum solvation model using eqn (9) in text.

${ }^{b}$ Aqueous $\mathrm{p}$ Kas calculated via the thermodynamic cycle (Figure 1 cycle C) using eqn (8) in text. 
Table S8. Aqueous free energies of enolization (in $\mathrm{kJ} \mathrm{mol}^{-1}$ ) calculated using various continuum solvation models.

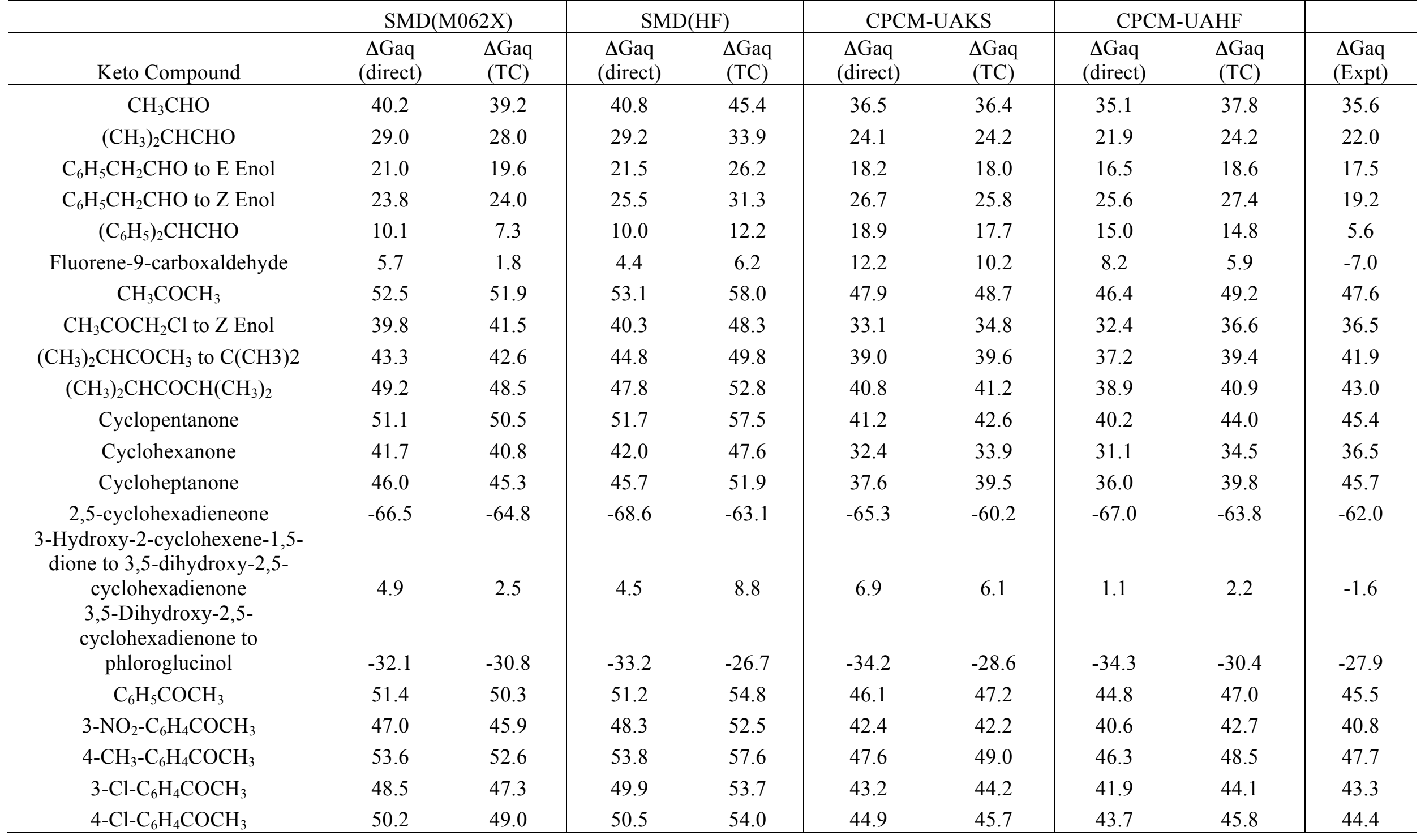




\begin{tabular}{|c|c|c|c|c|c|c|c|c|c|}
\hline $4-\mathrm{F}-\mathrm{C}_{6} \mathrm{H}_{4} \mathrm{COCH}_{3}$ & 52.2 & 51.1 & 52.0 & 55.8 & 46.9 & 47.8 & 45.4 & 47.6 & 46.3 \\
\hline $4-\mathrm{MeO}-\mathrm{C}_{6} \mathrm{H}_{4} \mathrm{COCH}_{3}$ & 55.7 & 54.8 & 55.8 & 59.9 & 51.0 & 52.5 & 49.9 & 52.0 & 50.3 \\
\hline $\mathrm{C}_{6} \mathrm{H}_{5} \mathrm{COCH}\left(\mathrm{CH}_{3}\right)_{2}$ & 42.4 & 41.5 & 40.8 & 44.1 & 36.4 & 37.0 & 36.2 & 37.0 & 37.0 \\
\hline acetylmesitylene & 39.4 & 38.3 & 38.2 & 47.2 & 35.0 & 36.1 & 35.5 & 37.3 & 39.5 \\
\hline 2-Indanone & 29.3 & 28.2 & 29.4 & 34.2 & 29.1 & 29.8 & 27.1 & 29.5 & 22.0 \\
\hline$\alpha$-3-Pyridylacetophenone & 33.5 & 30.8 & 33.3 & 38.0 & 24.1 & 24.0 & 24.1 & 25.8 & 27.5 \\
\hline Anthrone & 13.5 & 12.2 & 14.3 & 16.3 & 12.4 & 16.2 & 12.6 & 13.1 & 12.4 \\
\hline $\mathrm{CH}_{3} \mathrm{COOH}$ & 104.2 & 102.1 & 105.9 & 108.3 & 101.7 & 100.7 & 101.0 & 101.9 & 110.9 \\
\hline $\mathrm{CH}_{3} \mathrm{COOCH}_{3}$ & 117.3 & 115.3 & 118.2 & 120.5 & 112.4 & 111.9 & 111.1 & 112.1 & 110.9 \\
\hline Mandelic acid & 90.9 & 89.0 & 92.9 & 99.6 & 100.0 & 98.9 & 96.6 & 98.7 & 92.6 \\
\hline Meldrum's acid & 11.8 & 13.4 & 13.6 & 20.8 & 12.4 & 14.6 & 10.3 & 13.6 & 13.8 \\
\hline
\end{tabular}


Table S9. Free energies of hydrogen and chlorine atom transfer from chloroform to the carbon-centered radical (in kJ mol $\left.{ }^{-1}\right) .{ }^{a}$

\begin{tabular}{|c|c|c|c|c|}
\hline \multirow{2}{*}{ Radical } & \multicolumn{2}{|c|}{ HAT } & \multicolumn{2}{c|}{ CAT Direct } \\
\cline { 2 - 5 } & Direct & TC & -45.0 & -45.5 \\
\hline Methyl & -34.6 & -33.1 & -42.5 & -43.1 \\
\hline Ethyl & -16.9 & -15.4 & -41.6 & -42.3 \\
\hline Iso-propyl & 5.3 & 6.7 & -42.1 & -42.8 \\
\hline Tert-Butyl & 0.1 & 1.5 & -77.2 & -77.5 \\
\hline Cubyl & -36.1 & -35.0 & -68.1 & -68.7 \\
\hline Admantyl & -12.4 & -11.1 & & \\
\hline
\end{tabular}

${ }^{a}$ Calculations were carried out using the SMD solvation model and chloroform as solvent. 
Table S10. Calculated and experimental free energy barriers $\left(\mathrm{kJ} \mathrm{mol}^{-1}\right)$ of 18 Diels Alder reactions. ${ }^{a}$

\begin{tabular}{|c|c|c|c|c|c|c|}
\hline Solvent & Diene & Dienophile & TS & $\Delta \mathrm{G}^{\text {act }}$ (Direct) & $\Delta \mathrm{G}^{\mathrm{act}}(\mathrm{TC})$ & $\Delta \mathrm{G}^{\text {act }}(\operatorname{expt})^{b}$ \\
\hline benzene & & $\mathbf{1}$ & 1 & 105.1 & 104.9 & 110.9 \\
\hline benzene & 4 & 1 & 4 & 95.6 & 96.0 & 97.5 \\
\hline benzene & 7 & 1 & 7 & 82.2 & 82.9 & 91.2 \\
\hline benzene & 10 & 1 & 10 & 76.5 & 76.9 & 87.9 \\
\hline benzene & 12 & | & 12 & 73.5 & 73.9 & 69.9 \\
\hline acetonitrile & 15 & 15 & 15 & 95.2 & 96.1 & 100.8 \\
\hline water & 12 & 16 & 16 & 60.5 & 60.5 & 69.0 \\
\hline
\end{tabular}




\begin{tabular}{|c|c|c|c|c|c|c|}
\hline water & $\frac{41}{12}$ & 17 & 17 & 57.9 & 58.3 & 66.5 \\
\hline water & 22 & 17 & 22 & 70.1 & 71.4 & 77.4 \\
\hline water & 11 & 17 & 26 & 73.2 & 73.6 & 79.5 \\
\hline dioxane & 12 & 30 & 30 & 45.2 & 43.6 & 56.9 \\
\hline dioxane & & $\mathrm{ClOC}^{\prime}$ & 37 & 50.2 & 50.3 & 67.8 \\
\hline dioxane & $\frac{41}{12}$ & $\mathrm{MeO}_{2} \mathrm{C}=\frac{\overline{\overline{41}}}{\mathrm{CO}_{2} \mathrm{Me}}$ & 41 & 71.2 & 74.8 & 91.6 \\
\hline dioxane & 12 & 44 & 44 & 96.1 & 96.7 & 99.6 \\
\hline dioxane & $\frac{\square 1}{12}$ & $\begin{array}{c}\mathrm{CO}_{2} \mathrm{Me} \\
45\end{array}$ & 45 & 96.0 & 96.8 & 100.8 \\
\hline
\end{tabular}




dioxane

${ }^{a}$ The SMD-M062X solvent model was employed for these calculations. ${ }^{b}$ See Ref 21 for details. 
Table S11. Calculated free energy barriers $\left(\mathrm{kJ} \mathrm{mol}^{-1}\right)$ of 21 radical reactions

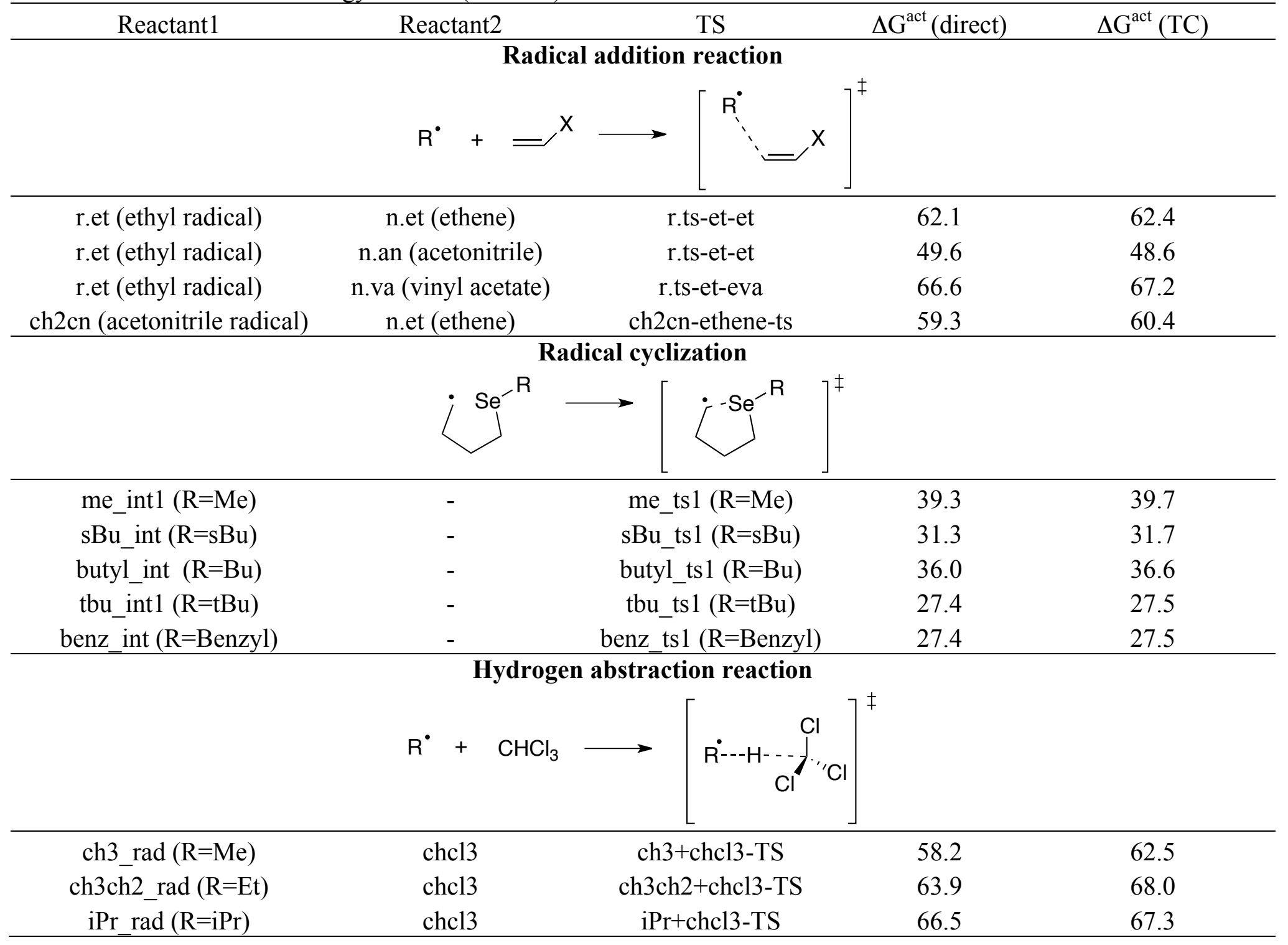




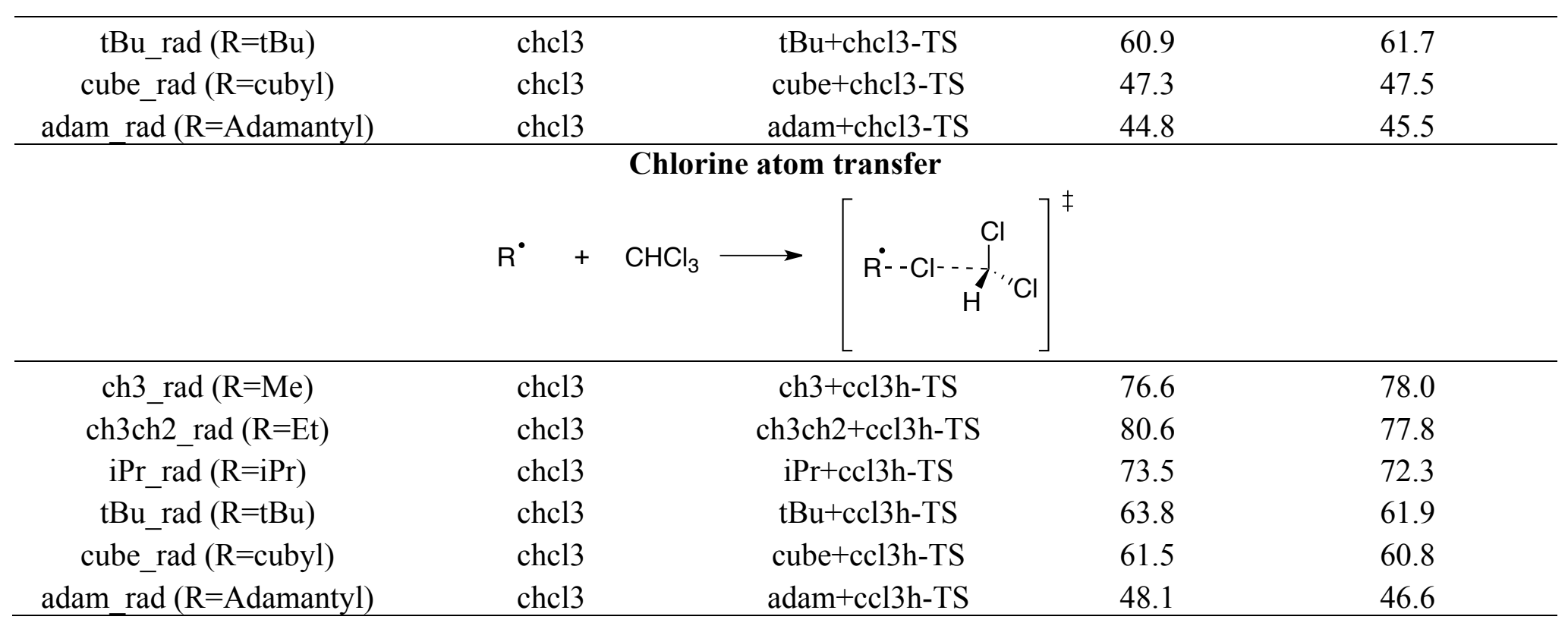

${ }^{a}$ The SMD-M062X solvent model was employed for these calculations 
Table S12. Calculated free energy barriers $\left(\mathrm{kJ} \mathrm{mol}^{-1}\right)$ of 45 ionic reactions. ${ }^{a}$

\begin{tabular}{|c|c|c|}
\hline SN2 reactions & $\Delta \mathrm{G}^{\text {act }}($ direct $)$ & $\Delta \mathrm{G}^{\mathrm{act}}(\mathrm{TC})$ \\
\hline $\mathrm{CN}(-)+\mathrm{EtCl}(\mathrm{DMSO})$ & 117.7 & 116.1 \\
\hline $\mathrm{CN}(-)+\mathrm{EtBr}(\mathrm{DMSO})$ & 117.7 & 116.1 \\
\hline CN(-)+EtI (DMSO) & 101.9 & 104.5 \\
\hline CN(-)+EtTosyl (DMSO) & 119.8 & 119.7 \\
\hline $\mathrm{Cl}(-)+\mathrm{MeCl}$ (Acetonitrile) & 100.1 & 99.3 \\
\hline $\mathrm{Cl}(-)+\mathrm{EtCl}$ (Acetonitrile) & 111.5 & 109.4 \\
\hline $\mathrm{Cl}(-)+\mathrm{iPrCl}$ (Acetonitrile) & 117.7 & 112.4 \\
\hline $\mathrm{Cl}(-)+\mathrm{PrCl}$ (Acetonitrile) & 109.8 & 107.4 \\
\hline $\mathrm{Cl}(-)+\mathrm{iBuCl}$ (Acetonitrile) & 117.3 & 114.2 \\
\hline $\mathrm{Cl}(-)+\mathrm{tBuCl}$ (Acetonitrile) & 120.6 & 113.5 \\
\hline $\mathrm{Cl}(-)+$ neopentylCl (Acetonitrile) & 143.4 & 139.2 \\
\hline $\mathrm{Cl}(-)+$ allylCl (Acetonitrile) & 97.0 & 93.6 \\
\hline cl(-)+cycpropcl (Acetonitrile) & 162.4 & 159.1 \\
\hline cl(-)+cycbutcl (Acetonitrile) & 124.0 & 118.7 \\
\hline cl(-)+cycpentcl (Acetonitrile) & 116.9 & 109.2 \\
\hline cl(-)+cychexcl (Acetonitrile) & 130.9 & 122.6 \\
\hline cl(-)+acetaldehyecl (Acetonitrile) & 83.3 & 81.8 \\
\hline $\mathrm{Cl}(-)+$ benzylCl (Acetonitrile) & 91.6 & 87.1 \\
\hline $\operatorname{cl}(-)+$ mebenzcl (Acetonitrile) & 101.4 & 91.1 \\
\hline $\mathrm{CN}(-)+\operatorname{mecl}($ Acetonitrile) & 100.4 & 99.8 \\
\hline CN(-)+etcl (Acetonitrile) & 119.7 & 118.1 \\
\hline CN(-)+iprcl (Acetonitrile) & 130.3 & 127.0 \\
\hline CN(-)+cycpropcl (Acetonitrile) & 170.9 & 168.3 \\
\hline CN(-)+cycbutcl (Acetonitrile) & 134.0 & 130.7 \\
\hline CN(-)+cycpentcl (Acetonitrile) & 128.8 & 124.3 \\
\hline CN(-)+cychexcl (Acetonitrile) & 142.8 & 137.2 \\
\hline CN(-)+allylcl (Acetonitrile) & 106.5 & 104.1 \\
\hline $\mathrm{CN}(-)+$ acetaldehyecl (Acetonitrile) & 88.4 & 87.4 \\
\hline $\mathrm{CN}(-)+$ mebenzcl (Acetonitrile) & 116.5 & 110.3 \\
\hline \multicolumn{3}{|l|}{$\mathrm{E} 2$ reactions } \\
\hline E2-cl(-)+etcl (Acetonitrile) & 149.8 & 142.3 \\
\hline E2-cl(-)+iprcl (Acetonitrile) & 133.0 & 124.5 \\
\hline E2-cl(-)+prcl (Acetonitrile) & 137.1 & 129.6 \\
\hline E2-cl(-)+tbucl (Acetonitrile) & 110.8 & 105.1 \\
\hline E2-cl(-)+cycpropcl (Acetonitrile) & 216.3 & 207.8 \\
\hline E2-cl(-)+cycbutcl (Acetonitrile) & 140.2 & 132.4 \\
\hline E2-cl(-)+cycpentcl (Acetonitrile) & 120.1 & 112.8 \\
\hline E2-cl(-)+cychexcl (Acetonitrile) & 124.7 & 116.2 \\
\hline E2-cl(-)+allylcl (Acetonitrile) & 179.3 & 172.7 \\
\hline E2-cl(-)+mebenzcl (Acetonitrile) & 119.4 & 113.3 \\
\hline
\end{tabular}

Michael addition 


\begin{tabular}{lll}
\hline MeS(-)+enone-1 (Water) & 35.1 & 35.2 \\
$M e S(-)+$ enone-2 (Water) & 57.2 & 54.0 \\
$M e S(-)+$ enone-3 (Water) & 44.6 & 44.2 \\
$M e S(-)+$ enone-4 (Water) & 45.7 & 45.4 \\
$M e S(-)+$ enone-5 (Water) & 50.9 & 52.1 \\
MeS(-)+enone-6 (Water) & 37.7 & 35.3 \\
\hline
\end{tabular}

${ }^{a}$ The SMD-M062X solvent model was employed for these calculations 